\title{
Interrelation between the Optical Conductivity and Crystal Field Splitting in Monolayer Manganites
}

\author{
K. Rościszewski and A.M. Oleś \\ M. Smoluchowski Institute of Physics, Jagiellonian University \\ Reymonta 4, PL-30-059 Kraków, Poland
}

\begin{abstract}
We introduce an effective model for correlated $e_{\mathrm{g}}$ electrons which reproduces qualitatively the evolution of magnetic order in monolayer manganites when correlated wave functions are used. Here we address recent optical conductivity measurements suggesting that the splitting between the occupied and empty $e_{\mathrm{g}}$ states is very large in $\mathrm{LaSrMnO}_{4}$, contrary to what is expected for the effective model. We argue that no contradiction was found but several simple-minded one-atom-based expectations concerning crystal-field splitting and optical conductivity are in error.
\end{abstract}

PACS numbers: 75.47.Lx, 75.10.Lp, 63.20.Pw

\section{Introduction}

The main difficulty in the theoretical description of perovskite manganese oxides is related to simultaneous importance of numerous degrees of freedom. Only in the last decade it has been recognized that the JahnTeller (JT) interactions and the superexchange, which occurs due to $e_{\mathrm{g}}$ electron excitations in the regime of large on-site Coulomb interactions, are both necessary to explain the onset of the orbital order [1] as well as to interpret [2] the experimental data including the optical properties of $\mathrm{LaMnO}_{3}$ [3]. In addition, it has also been realized that the core $t_{2 \mathrm{~g}}$ electrons play an essential role for the observed magnetic phases. Altogether, the observed magnetic order is a result of rather subtle balance between all these factors (see Ref. [4] and references therein).

The multi-band models and/or approaches based on the $a b$ initio or local density approximation (LDA) computations extended by static corrections due to local Coulomb interaction $U(\mathrm{LDA}+U)$, or the dynamical mean-field theory (LDA+DMFT) [5], provide the most sophisticated theoretical description of manganites nowadays. However, such calculations are in general quite costly and difficult to derive transparent conclusions. Thus, well motivated effective models are very helpful to identify the physical mechanisms responsible for particular types of order observed in the manganites.

\section{The model}

We adopt an effective two-dimensional (2D) model [6] featuring strongly correlated $e_{\mathrm{g}}$ electrons in undoped and half doped monolayer $\mathrm{La}_{1-x} \mathrm{Sr}_{1+x} \mathrm{MnO}_{4}$ manganites at
Mn sites, where the state of surrounding oxygens is included by an effective potential at each site. A realistic effective Hamiltonian which acts in the subspace of low energy $e_{\mathrm{g}}$ states could in principle be derived by an appropriate mapping of the results of $a b$ initio computations or starting from a multi-band model. A local basis at each manganese site is given by two Wannier orbitals of the $e_{\mathrm{g}}$ character (of $x^{2}-y^{2}$ and $3 z^{2}-r^{2}$ symmetry). We assume that the $t_{2 \mathrm{~g}}$ orbitals of $\mathrm{Mn}^{3+}$ ions are occupied by three "core" electrons with total spin $S=3 / 2$ these core electrons are treated as classical and frozen.

We use an effective Hamiltonian $\mathcal{H}$ for an $a b$ plane of a monolayer manganite

$$
\mathcal{H}=H_{\text {kin }}+H_{\text {cr }}+H_{\text {int }}+H_{\text {spin }}+H_{\mathrm{JT}}
$$

where the kinetic part $H_{\text {kin }}$ is expressed using the basis of two $e_{\mathrm{g}}$ orbitals

$$
x^{2}-y^{2} \sim|x\rangle, \quad 3 z^{2}-r^{2} \sim|z\rangle,
$$

or $\{x, z\}$ orbitals per site, with anisotropic phase dependent hopping,

$$
\begin{gathered}
H_{\mathrm{kin}}=-\frac{1}{4} t_{0} \sum_{\langle i j\rangle, \sigma}\left[3 d_{i x \sigma}^{\dagger} d_{j x \sigma}+d_{i z \sigma}^{\dagger} d_{j z \sigma}\right. \\
\left. \pm \sqrt{3}\left(d_{i x \sigma}^{\dagger} d_{j z \sigma}+d_{i z \sigma}^{\dagger} d_{j x \sigma}\right)+\text { h.c. }\right] .
\end{gathered}
$$

Here $d_{i \mu \sigma}^{\dagger}$ are creation operators for an electron at the $i$-th site, and $n$ - the corresponding number operators. The effective Mn-Mn hopping elements arise due to the hybridization with oxygen orbitals along $\mathrm{Mn}-\mathrm{O}-\mathrm{Mn}$ bonds, and are anisotropic and orbital dependent. The sum over $\langle i j\rangle$ runs over all pairs of nearest neighbors and the alternating sign \pm is interpreted as plus sign for $\langle i j\rangle$ being parallel to the crystal axis $a$ and minus for $\langle i j\rangle$ being parallel to the crystal axis $b$. The kinetic energy is 
supplemented by the crystal field term

$$
H_{\mathrm{cr}}=\frac{1}{2} E_{z} \sum_{i \sigma}\left(n_{i z \sigma}-n_{i x \sigma}\right),
$$

with $z$ orbital being favored for negative values of crystal field $E_{z}<0$.

The on-site electron interactions are given by

$$
\begin{aligned}
& H_{\mathrm{int}}=U \sum_{i \mu} n_{i \mu \uparrow} n_{i \mu \downarrow}+\left(U-\frac{5}{2} J_{\mathrm{H}}\right) \sum_{i} n_{i x} n_{i z} \\
& -\frac{1}{2} J_{\mathrm{H}} \sum_{i}\left(n_{i x \uparrow}-n_{i x \downarrow}\right)\left(n_{i z \uparrow}-n_{i z \downarrow}\right) \\
& -J_{\mathrm{H}} \sum_{i \mu} S_{i}^{z}\left(n_{i \mu \uparrow}-n_{i \mu \downarrow}\right),
\end{aligned}
$$

with the intraorbital Coulomb interaction element $U$, and Hund's exchange interaction $J_{\mathrm{H}}$. The interaction between $S=3 / 2$ spins on the bonds $\langle i j\rangle$,

$$
H_{\text {spin }}=J^{\prime} \sum_{\langle i j\rangle} S_{i}^{z} S_{j}^{z}
$$

stands for the antiferromagnetic (AF) $t_{2 \mathrm{~g}}$ superexchange $\left(J^{\prime}>0\right)$ between frozen core $t_{2 \mathrm{~g}}$ electrons [1]; here we keep again only the Ising part. Finally, the (simplified) form of the JT interactions,

$$
H_{\mathrm{JT}}=\sum_{i}\left[g\left(Q_{2 i} \tau_{i}^{x}+Q_{3 i} \tau_{i}^{z}\right)+\frac{1}{2} K\left(Q_{2 i}^{2}+Q_{3 i}^{2}\right)\right],
$$

includes the pseudospin $\tau_{i}^{\alpha} \equiv\left\{\tau_{i}^{x}, \tau_{i}^{z}\right\}$ operators (at site $i)$ :

$$
\begin{aligned}
\tau_{i}^{x} & =\sum_{\sigma}\left(d_{i x \sigma}^{\dagger} d_{i z \sigma}+d_{i z \sigma}^{\dagger} d_{i x \sigma}\right), \\
\tau_{i}^{z} & =\sum_{\sigma}\left(d_{i x \sigma}^{\dagger} d_{i x \sigma}-d_{i z \sigma}^{\dagger} d_{i z \sigma}\right) .
\end{aligned}
$$

The active JT deformation modes of the oxygens around a given $\mathrm{Mn}$ ion at site $i$ are labeled as $\left\{Q_{2 i}, Q_{3 i}\right\}$ - they lift out the degeneracy of $e_{\mathrm{g}}$ orbitals.

We use the standard parameters for the numerical calculations, motivated by the previous studies, as discussed in detail in Ref. [6]. Thus we take an effective $(d d \sigma)$ hopping element $t=0.4 \mathrm{eV}$. The effective on-site Coulomb repulsion was fixed as $U=7 t$, and for Hund's exchange we take $J_{H}=0.5 \mathrm{eV}[3]$. For the JT parameters adopted $K=13 \mathrm{eV} / \AA^{2}$ and $g=3.8 \mathrm{eV} / \AA$ and $J^{\prime}=3.0 \mathrm{meV}$ for the superexchange [4]. We consider the crystal field splitting $E_{z}$ in the range from 0 up to $-0.4 \mathrm{eV}$ motivated by experiment and by ab initio computations.

We study 2D square clusters (containing $N=64$ sites) with periodic boundary conditions, undoped and with hole doping $x=0.5$. First, the calculations within the single-determinant Hartree-Fock (HF) approximation were performed to determine the ground state wave function $\left|\Psi_{\mathrm{HF}}\right\rangle$. In the next step the $\mathrm{HF}$ wave function was improved using an exponential local ansatz which leads to the correlated ground state. For technical details see Refs. [6, 7].

The computations were run starting from several (different) initial conditions, i.e., from predefined charge, spin configuration, predefined pattern of core spins and a predefined set of lattice distortions $\left\{Q_{2 i}, Q_{3 i}\right\}$. Finally, the resulting set of energies (different locally stable states) was inspected and the lowest energy state was identified as a good candidate for the true ground state.

\section{Optical conductivity}

The Hamiltonian parameters used for the model computations reproduced the phase situation in $\mathrm{La}_{1-x} \mathrm{Sr}_{1+x} \mathrm{MnO}_{4}$ reasonably well [6]. One finds a $G$-type AF phase for the undoped compound, and the checkerboard charge order for the half-doped one, which can be accompanied by the zig-zag CE magnetic order when the proper JT distortions stabilize it. However, quite recent data based upon optical conductivity measurements in undoped $\mathrm{LaSrMnO}_{4}$ [8] provided some basic results which seemingly contradict the commonly accepted effective model parameters for manganites. First of all, multipeak structure was observed in optical conductivity with strongest peaks at $\approx 5.5 \mathrm{eV}$. Then, working in the framework of multi-band model (featuring true oxygen and manganese ions) but also making direct references to the effective model and fitting the optical data the authors estimated that the distance between (hypothetically nonsplit) $t_{2 \mathrm{~g}}$ and $e_{\mathrm{g}}$ levels should be $1.2 \mathrm{eV}$, and that the splitting within $t_{2 \mathrm{~g}}$ group is $0.1 \mathrm{eV}$ and splitting between two $e_{\mathrm{g}}$ levels (i.e. $x$ and $z$ orbitals) is $1.4 \mathrm{eV}$. The latter huge value was a great surprise. Up to now it was expected that the values of 0 to $-0.4 \mathrm{eV}$ should correspond to the effective model.

Before going towards too hasty conclusions we notice that it is difficult to deduce from the paper [8] if the values provided are generic for $\mathrm{Mn}$ ions in the framework of a multi-band (manganese+oxygen) model, i.e., if they are bare values (on Mn ions), or if they could still apply to the effective single site model such as Eq. (1) (screened values), or, finally, if they are simply values describing in a heuristic way the results of the optical experiments (i.e., without strict inter-relation to specific model). To clarify the situation one should simply compute the Green function related to the optical conductivity. This however would be rather involved for such a complicated model as that given by Eq. (1). To provide a cheaper alternative we asked ourselves the question which value would provide the optical experiment performed on a hypothetical single $\mathrm{Mn}$ ion (immersed in crystal field) with $t_{2 \mathrm{~g}}$ core fully occupied and one single electron in the lower $e_{\mathrm{g}}$ orbital. The answer seems obvious - the optical peak would be equal to the crystal field splitting $0.4 \mathrm{eV}$ of $e_{\mathrm{g}}$ orbitals if the effective model is right, or $1.4 \mathrm{eV}$ as suggested by Ref. [8]. Then we asked the question if the optical transition occurring on the same effective Mn ion but in presence of all the other lattice sites would provide the same value (or something quite similar)? If the answer was indeed positive, then we were in trouble and the common know-how concerning the values of effective models crystal splitting should be revised. In fact, the answer was negative, i.e., it was rather counterintuitive. 
TABLE

Energy of local excitation $\Delta$ versus crystal-field splitting $E_{z}$ (both in $[\mathrm{eV}]$ ) for $\mathrm{La}_{1-x} \mathrm{Sr}_{1+x} \mathrm{MnO}_{4}$. Legend: $x=0-{ }^{a} \mathrm{G}$-AF phase with uniform charges; $x=0.5$ $-{ }^{b}$ ferromagnetic phase, ${ }^{c} \mathrm{C}$-type $\mathrm{AF}$ phase, ${ }^{d} \mathrm{CE}$ phase, all with checkerboard charge order.

\begin{tabular}{c|l|c|c}
\hline \hline$E_{z}$ & 0.0 & -0.2 & -0.4 \\
\hline$\Delta(x=0.0, U=7 t)$ & $4.64^{a}$ & 5.15 & 5.26 \\
$\Delta(x=0.0, U=9 t)$ & 5.43 & 5.99 & 6.10 \\
$\Delta(x=0.5, U=7 t)$ & $1.66^{b}$ & $1.30^{c}$ & $1.50^{d}$
\end{tabular}

To be more precise, we picked up the sites with the number of $e_{\mathrm{g}}$ electrons (in the ground state) being close to 1 (in the undoped and half-doped cluster separately). Then (instead of Green function) we computed a matrix element

$$
\Delta=\frac{\left\langle\Psi_{\mathrm{HF}}\left|\left(d_{2}^{\dagger} d_{1}\right)^{\dagger}\left(H_{\mathrm{HF}}-E_{\mathrm{HF}}\right)\left(d_{2}^{\dagger} d_{1}\right)\right| \Psi_{\mathrm{HF}}\right\rangle}{\left\langle\Psi_{\mathrm{HF}}\left|\left(d_{2}^{\dagger} d_{1}\right)^{\dagger}\left(d_{2}^{\dagger} d_{1}\right)\right| \Psi_{\mathrm{HF}}\right\rangle},
$$

using the HF ground state $\left|\Psi_{\mathrm{HF}}\right\rangle$ (no correlations). We assumed here that index 1 stands (as a short notation) for site $i$, up spin and occupied $e_{\mathrm{g}}$ orbital together (by occupied $e_{\mathrm{g}}$ orbital we mean a linear combination of $x$ and $z$ orbitals, as found in the ground state computations for the $i$-th site). Similarly, index 2 stands for the same lattice site $i$, the same up spin, and the unoccupied $e_{\mathrm{g}}$ orbital (being a linear combination orthogonal to that corresponding to the occupied orbital). Because we picked the site with the occupation number close to 1 , the value of $\Delta$ equals approximately the energy of the optical excitation occurring on the same site. This excitation should be the only one detected in the optical conductivity measurements performed parallel to the crystal $c$ axis and the strongest peak for measurements performed along $a$ or $b$ axes (i.e., optical conductivity parallel to the single layer). The experimental result for this peak (for the undoped phase) is $\approx 5.5 \mathrm{eV}$ [8] and this is remarkably well reproduced by our computations - see entries in Table. We notice that $\Delta=5.26 \mathrm{eV}$ corresponding to $E_{z}=-0.4 \mathrm{eV}$ and $U=7 t$ is very close indeed to $5.6 \mathrm{eV}$. The lower row entries do suggest that $U / t$ could be slightly larger in the effective model — a value between 7 and 8 . Then the agreement between $\Delta$ and the experimental peaks would be even better. Thus we conclude that the dependence of the optical peak on crystal field splitting $E_{z}$ is weak - site occupations, the type of order and the Coulomb repulsion $U$ seem to play here the dominant role.

As an extra test we performed similar computations for the doped $x=0.5$ compound with checkerboard type charge order (with magnetic order depending on $E_{z}$ ). The motivation was that the optical transitions occurs on site with total charge close to unity (not strictly 1 therefore less accuracy) while the nearest-neighbor sites are almost empty. Thus the physical situation is now much closer to our imaginary experiment performed on the system with a single ion. Even in this case we do not obtain something close to $E_{z}$. Still the last row in Table demonstrates that $\Delta(x=0.5)$ is now much smaller. It does not evolve smoothly with $E_{z}$ but this is to be expected as the magnetic order changes and the $x$ and $z$ orbital occupation varies with $E_{z}$. However, the $\Delta=1.5 \mathrm{eV}$ value (for $x=0.5$ and $E_{z}=-0.4 \mathrm{eV}$ ) is very close to the value $1.4 \mathrm{eV}$ which the authors of [8] claim to be their crystal field splitting. This could be more than a coincidence, but we cannot draw any definite conclusion as we have very limited information about the crystal field splitting and its meaning in Ref. [8].

\section{Summary}

We conclude that the effective model used up to now [6] performs quite well even for the description of non-static phenomena such as optical conductivity. We also found that in strongly correlated manganite systems (with one occupied $e_{\mathrm{g}}$ level) the crystal field splitting between occupied and unoccupied electron levels does not directly translate into optical conductivity peaks.

\section{Acknowledgments}

A.M. Oleś acknowledges support by the Foundation for Polish Science (FNP). This work was supported by the Polish Ministry of Science and Education under project No. N202 068 32/1481.

\section{References}

[1] L.F. Feiner, A.M. Oleś, Phys. Rev. B 59, 3295 (1999).

[2] A.M. Oleś, P. Horsch, L.F. Feiner, G. Khaliullin, Phys. Rev. B 72, 214431 (2005).

[3] N.N. Kovaleva, A.V. Boris, C. Bernhard, A. Kulakov, A. Pimenov, A.M. Balbashov, G. Khaliullin, B. Keimer, Phys. Rev. Lett. 93, 147204 (2004).

[4] M. Daghofer, W. von der Linden, A.M. Oleś, Phys. Rev. B 70, 184430 (2004).

[5] A. Yamasaki, M. Feldbacher, Y.-F. Yang, O.K. Andersen, K. Held, Phys. Rev. Lett. 96, 166401 (2006).

[6] K. Rościszewski, A.M. Oleś, J. Phys., Condens. Matter 19, 186223 (2007).

[7] D. Góra, K. Rościszewski, A.M. Oleś, Phys. Rev. B 60, 7429 (1999).

[8] A. Gössling, M.W. Haverkort, M. Benomar, H. Wu, D. Senff, T. Möller, M. Braden, J.A. Mydosh, M. Grüninger, Phys. Rev. B 77, 035109 (2008). 